\title{
LOS MORISCOS QUE PERMANECIERON EN EL OBISPADO DE ORIHUELA DESPUÉS DE 1609
}

Por

ROSA MARIA BLASCO MARTÍNEZ

La permanencia de la población morisca en tierra peninsulares tras el decreto de expulsión fue mínima, insignificante; no podía ser de otra forma dada la voluntad política de arrancar de raiz los restos islámicos que aún quedaban en España, y la eficacia burocrática con que se llevó a cabo la expulsión entre 1609 y 1614 , como puso de manifiesto $H$. Lapeyre.

No obstante, el tema de los moriscos que se quedaron despertó muy pronto el interés de la historiografía (1) y no ha estado ausente de las obras más recientes, aunque el tema no se haya tratado con la intensidad y la reiteración que otros relacionados con la cuestión morisca (2).

El obispo de Orihuela, Fr. Andrés Balaguer, en carta a Fr. Antonio Sobrino de 30 de septiembre de 1609, se quejaba de la marcha generalizada de moriscos en algunos lugares de su obispado: "las casas de Petrel se fueron todas sino dos que el conde de Elda mandó quedar por fuerça (...); en Monóvar que es de 250 vezinos, quedan 30 de estos medio voluntarios. En Albatera, lugar de 300 vezinos, quedan 36 casas; las casas de Elche son 39 pero todos se quieren ir $y$ el señor duque los tiene por fuerça» (3).

Sin embargo el mismo prelado, pocos meses después, confirma la per-

(1) F. FERNÁNDEZ Y GONZÁLEZ, «De los moriscos que permanecieron en España después de la expulsión decretada por Felipe lil», en Revista de España XIX (1871), pp. 103-114, y XX (1871), pp. 363-376.

(2) Cfr. la obra de síntesis de A. DOMINGUEZ ORTIZ y B. VINCENT, Historia de los moriscos. Vida y tragedia de una minoria, Madrid 1978, pp. 247-266.

(3) Citado en M. MARTÍNEZ GOMIS, "EI problema morisco en tierras alicantinas, 1520-1614», Historia de la provincia de Alicante, p. 362 . 
manencia de moriscos en un memorial que dirige al rey, el 10 de marzo de 1610: "... en Alicante y toda su huerta quedan muchos moriscos, assi pequeños como grandes, assi hombres como mujeres, de 20, 30, 40 y 50 años, los quales les han trahydo como esclavos y los tienen por tales" (4).

El propio monarca encarga al obispo Balaguer la valoración de la población morisca que permanece; como respuesta he localizado un informe del prelado, fechado el 25 de agosto de 1610 (5), donde relata pormenorizadamente los que se han quedado, cifra muy escasa tanto si se compara con la anterior población morisca como respecto a los cristianos viejos del obispado. necen:

En su relación clasifica en cuatro apartados a los moriscos que perma-

1. Los niños moriscos; en este grupo incluye también a algunas personas mayores que permanecen en el reino sin licencia. Suman 249 , aunque el documento da la cifra de 248.

2. Los moriscos que tienen licencia del obispado por haber dado muestras de ser buenos cristianos desde antes del decreto de expulsión. Suman 47.

3. Los moriscos que han probado ser hijos de cristianos viejos y pretenden, por tanto, que no les afectan los bandos de la expulsión. Son 7.

4. Las moriscas casadas con cristianos viejos o que han quedado viudas. Son 4 .

En el conjunto de los cuatro apartados se computan 306 personas, que en su mayoría son niños.

La práctica de poner a niños y niñas moriscos bajo el cuidado de familias cristianas se había dado ya durante la rebelión granadina de la segunda mitad del S. XVI (6), y volvió a producirse de nuevo en las fechas en que tuvo lugar la expulsión, entre 1609 y 1614.

El tema de los niños moriscos del reino de Valencia ha suscitado el interés de diversos autores (7).

(4) P. BORONAT, Los moriscos españoles y su expulsión. Estudio histórico critico. Volúmenes. Valencia, 1901. Vol. II, Apéndice documental, p. 585.

(5) Relación de los moriscos quue quedan en el obispado de Orihuela hecha por mandato de S. M. por el obispo Cfr. Andrés Balaguer a 25 de agosto de 1610. Archivo General de Simancas, Estado, Ig. 224.

(6) A. CABRILLANA, "Almería en el S. XVI: moriscos encomendados", en Revista de Archivos, Bibliotecas y Museos, enero-junio 1975, pp. 41-68; R. BENÍTEZ SÁNCHEZ-BLANCO, "Guerra y sociedad: Málaga y los cautivos, 1569", en Estudis, III (1974), pp. 31-54.

(7) V. CASTANEDA, «Manifestación de los hijos de moriscos que quedaron en la villa de Onteniente al verificarse la expulsión de éstos del reino de Valencia, 1611 ", en Boletín de la Real Academia de la Historia, LXXXII (1923), pp. 421-427; M. BARCELÓ, "Els nins moriscos", en Primer Congreso de Historia del País Valenciano, Valencia, 1975, III, pp. 327-331; M. MARTÍNEZ GOMIS, «El control de los niños moriscos en Alicante tras el decreto de expulsión de 1609", en Anales de la Universidad de Alicante. Historia Moderna, n. ${ }^{\circ} 1(1981)$, pp. 251-280. 
El Informe del obispo Balaguer, que se había comprometido a tomar bajo su protección a los niños moriscos de su diócesis (8), se corresponde cronológicamente con los datos que aporta Mario Martínez Gomis en su artículo sobre Alicante - el que llama registro $n .^{\circ} 1-$, pero aquél extiende la información a todo el ámbito del obispado.

Efectúa el registro de los niños por poblaciones, anotando de forma sistemática primero a las niñas y a continuación a los niños, ordenados según criterio de edad: comienza por los más pequeños y termina por los mayores.

Presentamos los datos de forma esquemática para una mejor comprensión y valoración de esta información.

ORIHUELA Varones: 12

Hembras: $9 \quad$ Total: 21

\begin{tabular}{|c|c|c|c|}
\hline EDADES & V. & $\mathrm{H}$. & $\mathrm{T}$. \\
\hline 8 meses & 1 & - & 1 \\
\hline 1 año & - & 1 & 1 \\
\hline 3 años & 1 & 1 & 2 \\
\hline 4 años & 1 & - & 1 \\
\hline 5 años & - & 2 & 2 \\
\hline 7 años & 1 & 1 & 2 \\
\hline 8 años & 3 & 2 & 5 \\
\hline 9 años & 2 & - & 2 \\
\hline 10 años & 1 & 1 & 2 \\
\hline 12 años & 2 & 1 & 3 \\
\hline
\end{tabular}

CALLOSA Varón: $\quad 1 \quad 10$ años

ALBATERA Varones: 2

Hembras: 3

\begin{tabular}{l} 
EDADES \\
\hline 8 meses \\
4 años \\
5 años \\
8 años \\
9 año
\end{tabular}

\begin{tabular}{c}
$\mathrm{V}$ \\
\hline 1 \\
$\frac{1}{1}$ \\
-
\end{tabular}

Total: 5

(8) El Consejo de Estado había autorizado su permanencia responsabilizando al obispo de su seguimiento, sobre todo en lo concerniente a una educación religiosa cristiana y a evitar todo tipo de contactos con sus padres y familiares. Cfr. J. B. VILAR, "Los moriscos de la gobernación y obispado de Orihuelan en Al-Andalus, XLIII (1976), pp. 323-367, especialmente p. 358. 
REDOVÁN

Varón: $1 \quad 4$ años

CATRAL

Varón: 11 año

ELCHE

Varones: 2

Hembras: 8 Total: 10

\begin{tabular}{|c|c|c|c|}
\hline EDADES & V. & $\mathrm{H}$. & T. \\
\hline 8 meses & - & - & - \\
\hline 2 años & - & 2 & 2 \\
\hline 4 años & - & 2 & 2 \\
\hline 7 años & 2 & 1 & 3 \\
\hline 10 años & - & 2 & 2 \\
\hline
\end{tabular}

CREVILLENTE

Varones: 1

Hembras: 1

Total: 2

\begin{tabular}{l} 
EDADES \\
\hline 6 años \\
9 años
\end{tabular}
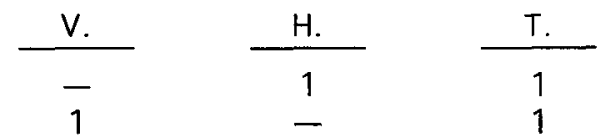

ASPE

Varones: 5

Hembras: 2 Total: 7

\begin{tabular}{r} 
EDADES \\
\hline 4 años \\
5 años \\
9 años \\
10 años \\
12 años
\end{tabular}
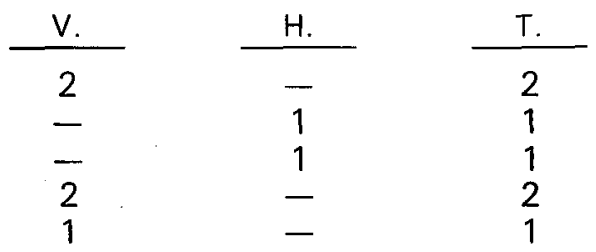


\section{ALICANTE}

En la colegial de San Nicolás.

Varones: 26

Hembras: 52

Total: 78

\begin{tabular}{c} 
EDADES \\
\hline 8 meses \\
2 años \\
3 años \\
4 años \\
5 años \\
6 años \\
7 años \\
8 años \\
9 años \\
10 años \\
12 años \\
13 años \\
33 años
\end{tabular}

\begin{tabular}{c}
$\mathrm{v}$. \\
\hline 1 \\
2 \\
4 \\
6 \\
1 \\
3 \\
9 \\
- \\
- \\
- \\
- \\
-
\end{tabular}

\begin{tabular}{c} 
H. \\
\hline- \\
2 \\
4 \\
7 \\
9 \\
5 \\
8 \\
6 \\
1 \\
6 \\
2 \\
1 \\
1
\end{tabular}

\begin{tabular}{r} 
T. \\
\hline 1 \\
4 \\
8 \\
13 \\
10 \\
8 \\
17 \\
6 \\
1 \\
6 \\
2 \\
1 \\
1
\end{tabular}

En la colegial de Santa María.

Varones: 9

Hembras: 23

Total: 32

\begin{tabular}{c} 
EDADES \\
\hline 4 años \\
5 años \\
6 años \\
7 años \\
8 años \\
9 años \\
10 años \\
12 años \\
20 años \\
30 años
\end{tabular}

\begin{tabular}{c}
$\mathrm{V}$ \\
\hline$\frac{1}{1}$ \\
2 \\
$\frac{1}{1}$ \\
$\frac{1}{3}$ \\
-
\end{tabular}

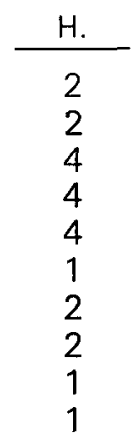

\begin{tabular}{c}
$\mathrm{T}$. \\
\hline 3 \\
2 \\
5 \\
6 \\
5 \\
1 \\
3 \\
5 \\
1 \\
1
\end{tabular}

SAN JUAN Y BENIMAGRELL

Varones: 18

Hembras: 9

Total: 27 


\begin{tabular}{c} 
EDADES \\
\hline 8 meses \\
2 años \\
3 años \\
4 años \\
5 años \\
7 años \\
8 años \\
10 años \\
11 años \\
12 años \\
24 años \\
46 años
\end{tabular}

\begin{tabular}{c}
$\mathrm{v}$ \\
\hline 1 \\
$\frac{1}{1}$ \\
3 \\
2 \\
3 \\
2 \\
4 \\
-1 \\
1 \\
-
\end{tabular}

BUSOT

Varones: 2

Hembras:
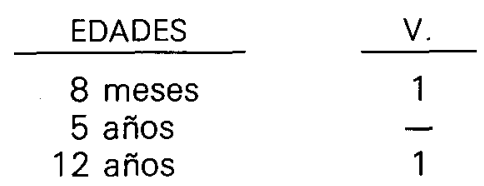

Total: 3
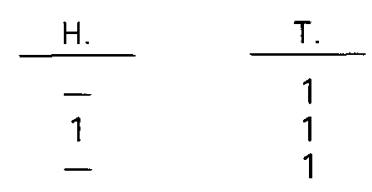

MUCHAMIEL

Varones: 12

Hembras: 12

Total: 24

\begin{tabular}{c} 
EDADES \\
\hline 2 años \\
3 años \\
4 años \\
5 años \\
6 años \\
7 años \\
8 años \\
9 años \\
10 años \\
12 años \\
14 años
\end{tabular}

\begin{tabular}{c}
$\mathrm{v}$ \\
\hline$-\overline{3}$ \\
$\frac{2}{2}$ \\
1 \\
2 \\
1 \\
$\overline{1}$ \\
2 \\
-
\end{tabular}

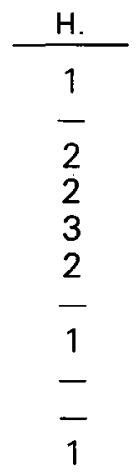

\begin{tabular}{l}
$\mathrm{T}$. \\
\hline 1 \\
3 \\
2 \\
4 \\
4 \\
4 \\
1 \\
1 \\
1 \\
2 \\
1
\end{tabular}


VILLAFRANQUEZA

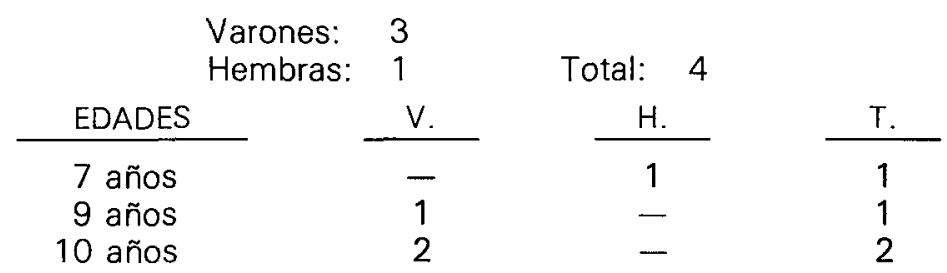

ELDA

Varones: 2

Edades: 8 años 1

14 años 1

PETREL

Varones: 1

Hembras: 1 Total: 2

EDADES
7 años
30 años
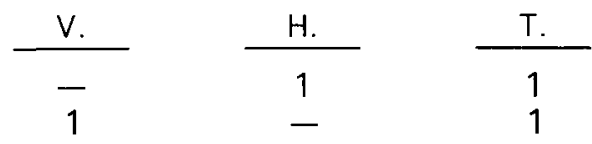

NOVELDA

Varones: 4 Total: 4

\begin{aligned} & \multicolumn{2}{c}{ EDADES } \\ & \hline 4 años 1 \\ & 9 años 1 \\ & 12 años 1 \\ & 18 años 1\end{aligned}

MONÓVAR

Hembra: 14 años

CAUDETE

Varones: $3 \quad$ Total: 3

Edades: 4 años 2

10 años 1 
AYORA

Varones: 9

Hembras: 12

Total: 21

\begin{tabular}{|c|c|c|c|}
\hline EDADES & V. & $\mathrm{H}$. & $\mathrm{T}$. \\
\hline 8 meses & 1 & - & 1 \\
\hline 1 año & - & 1 & $i$ \\
\hline 2 años & 1 & - & 1 \\
\hline 3 años & 1 & 1 & 2 \\
\hline 4 años & - & 4 & 4 \\
\hline 5 años & - & 2 & 2 \\
\hline 7 años & 2 & - & 2 \\
\hline 8 años & 1 & - & 1 \\
\hline 10 años & 2 & 2 & 4 \\
\hline 11 años & 1 & 2 & 3 \\
\hline
\end{tabular}

De este grupo todos tienen licencia para permanecer excepto siete personas mayores que carecen de ella. Son:

Alicante. En la iglesia colegial, Ángela de 33 años, en casa del caballero Jerónimo Vallebrera. En la parroquia de Sta. María, Ángela, de 20 años, en casa del baile Pedro Martínez de Vera, y Esperanza, de 30 años, en casa de la viuda del caballero Vicente Martínez.

San Juan: Ángela, de 46 años, en el monasterio de la Verónica o Santa Faz, y Juan, de 24 años, en el mismo lugar.

Petrel: Francisco Amoret y García, de 30 años, casado con cristiana vieja.

Novelda: Luis, de 18 años; lo llevó a Valencia su amo.

Todos parecen ser moriscos y no deben confundirse con esclavos berberiscos.

Los 242 restantes son niños moriscos con licencia para quedarse.

Al tratarse del año siguiente a la expulsión, tan sólo dos de ellos alcanzan los 13 y 14 años.

En conjunto suponen: Varones: 111

Hembras: 131

Entre 0 y 6 años: 116

Entre 7 y 14 años: 126

Total: 242

Predominan las niñas frente a los niños; también es superior el número 
de los mayores de 7 años, edad que permite a los niños colaborar con algún tipo de ayuda o prestación en la familia que los acoge.

Otro dato a destacar es que el $44,2 \%$ de todos los niños moriscos del obispado de Orihuela están en Alicante.

Entre la fecha de la Relación del obispo y la de la fuente que Martínez Gomis utiliza para estudiar a los niños moriscos de Alicante, que llama registro número 1 (9), media tan sólo un mes de diferencia; pero, pese a la proximidad cronológica de estas dos fuentes, los datos que aportan están lejos de ser coincidentes.

$\frac{\text { REGISTRO N. }{ }^{\circ} 1}{16-V I I-1610} \quad \frac{\text { RELACIÓN OBISPO ORIHUELA }}{25-\mathrm{VIII-1610}}$

\begin{tabular}{|c|c|c|c|c|c|c|}
\hline EDADES & v. & $\mathrm{H}$. & $\mathrm{T}$. & $\mathrm{V}$. & $\mathrm{H}$. & $\mathrm{T}$. \\
\hline Inferior al año & 1 & - & 1 & 1 & - & 1 \\
\hline 1 año & 1 & 1 & 2 & - & - & - \\
\hline 2 años & 1 & 1 & 2 & 2 & 2 & 4 \\
\hline 3 años & 2 & 3 & 5 & 4 & $\overline{4}$ & 8 \\
\hline 4 años & $\overline{5}$ & 6 & 11 & 7 & 9 & 16 \\
\hline 5 años & 4 & 7 & 11 & 1 & 11 & 12 \\
\hline 6 años & 3 & 5 & 8 & 4 & 9 & 13 \\
\hline 7 años & 12 & 11 & 23 & 11 & 12 & 23 \\
\hline 8 años & 1 & 14 & 15 & 1 & 10 & 11 \\
\hline 9 años & 1 & 2 & 3 & - & 2 & 2 \\
\hline 10 años & - & 3 & 3 & 1 & 8 & 9 \\
\hline 11 años & - & 3 & 3 & - & - & $\ldots$ \\
\hline 12 años & - & 1 & 1 & 3 & 4 & 7 \\
\hline 13 años & - & - & - & - & 1 & 1 \\
\hline Sin especificar & 4 & - & 4 & - & - & 二 \\
\hline Totales & 31 & 57 & 88 & 55 & 72 & 107 \\
\hline
\end{tabular}

En primer lugar el número de niños moriscos es notablemente superior en los datos que aporta el obispo: 107 frente a los 88 del registro número 1. En cambio, la cifra que da el obispo Balaguer se aproxima más a la que Martínez Gomis recoge en el registro número 2, que responde al sondeo realizado un año después.

Este autor atribuye la diferencia que se advierte entre el primero y segundo registro a la ocultación de los niños varones a partir de los 8 años de edad.

Sin embargo, de los 19 asientos de más que refleja el informe Balaguer, 15 corresponden a niñas.

(9) M. MARTINEZZ GOMIS, art. cit., p. 266. 
Por otra parte, es el grupo radicado en Alicante el que ofrece un marcado desequilibrio entre los dos sexos, a favor de las hembras, mientras en el conjunto de las otras poblaciones los niños (78) superan a las niñas (60).

Los lugares de procedencia de los moriscos del obispado de Orihuela quedan reflejados en el siguiente cuadro. 
LOS MORISCOS QUE PERMANECIERON EN EL OBISPADO... / BlasCo

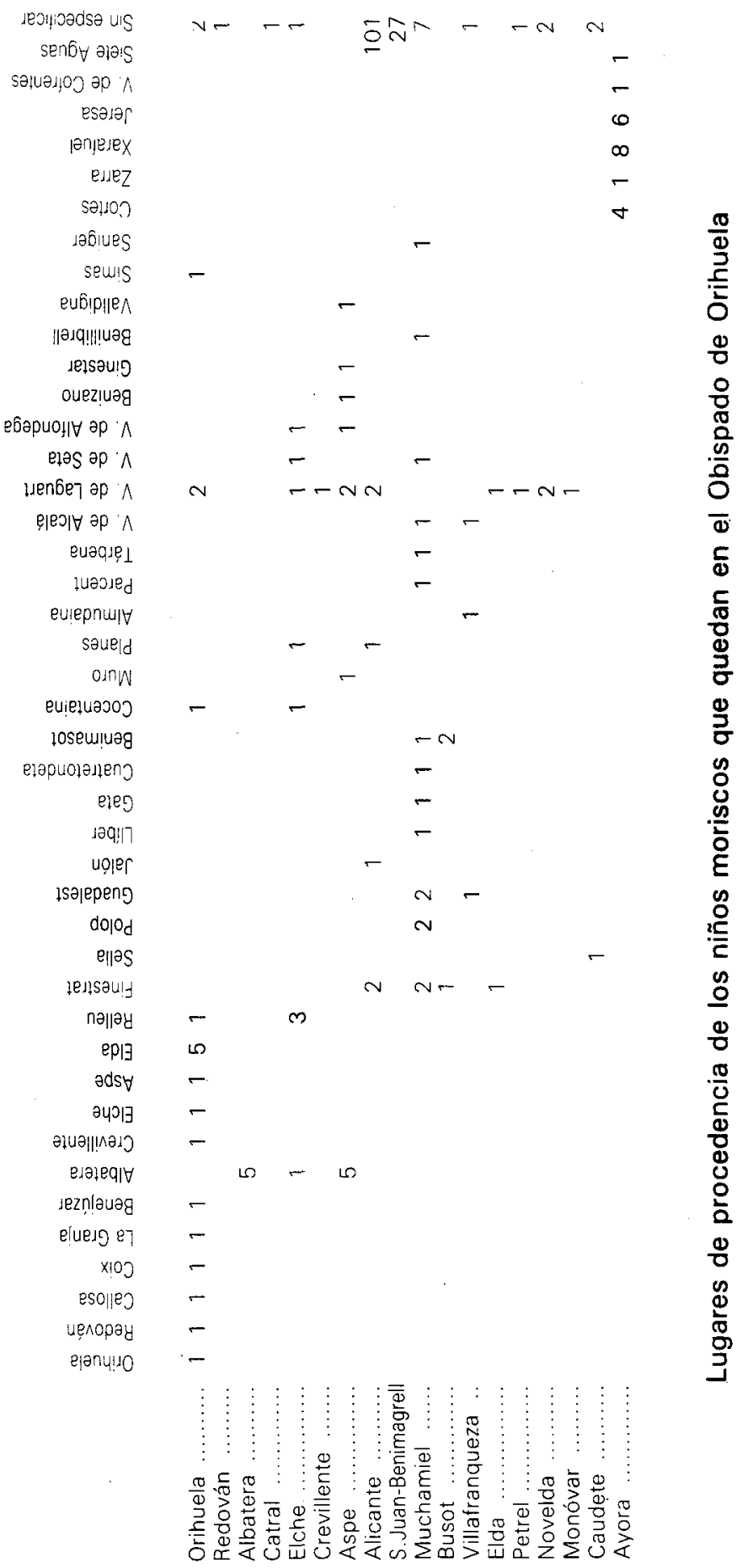


En lo referente a la procedencia de estos moriscos, el informe del obispo no la indica para Alicante - salvo en 6 casos-, San Juan y Benimagrell.

Martínez Gomis en su estudio pudo establecer la procedencia de 71 niños moriscos de Alicante: 50 eran originarios de los lugares que se habían hecho fuertes en Val de Laguart; 4 de los núcleos cuya población se había atrincherado en la Muela de Cortes; por último otros 9 procedían de lugares que no ofrecieron resistencia alguna a la expulsión (10).

Los núcleos de San Juan y Benimagrell también intervinieron en la represión de los moriscos resistentes en Val de Laguart; de donde se puede suponer que los 27 niños moriscos registrados por el obispo Balaguer procederían de los lugares cuyos vecinos se habian refugiado en dicho valle.

Si se observa el cuadro, buena parte de los moriscos de Elche, Aspe, Muchamiel, Villafranqueza, Elda, Petrel, Novelda, Monóvar y Caudette proceden asimismo de lugares invọlucrados en la revuelta de Val de Laguart.

Los asentados en Ayora son originarios de poblaciones próximas de la actual provincia de Valencia; la mayoría de sus vecinos se habían concentrado en la Muela de Cortes - actual provincia de Albacete- de donde fueron desalojados por el tercio de Lombardía.

La mayoría, por tanto, eran botín de guerra.

Queda constancia de que una de las niñas, procedente de Xarafuel, fue entregada por su madre al boticario, ante escribano.

Un número notablemente inferior procede de poblaciones que no habían ofrecido resistencia ante el decreto de expulsión.

En cuanto a los grupos sociales receptores de estos niños moriscos, se pueden sintetizar en el siguiente cuadro. Recoge el número de niños registrados en casa del clero, caballeros, ciudadanos, cargos de justicia y municipales, personas con el título de «don», médicos cirujanos y boticarios, mercaderes, notarios, oficios agremiados, albañil y mesonero, labrador. Se indica, por último, la cifra de receptores de quienes no se especifica oficio ni categoría social.

(10) Ibidem, p. 273 
LOS MORISCOS QUE PERMANECIERON EN EL OBISPADO... / BlasCo
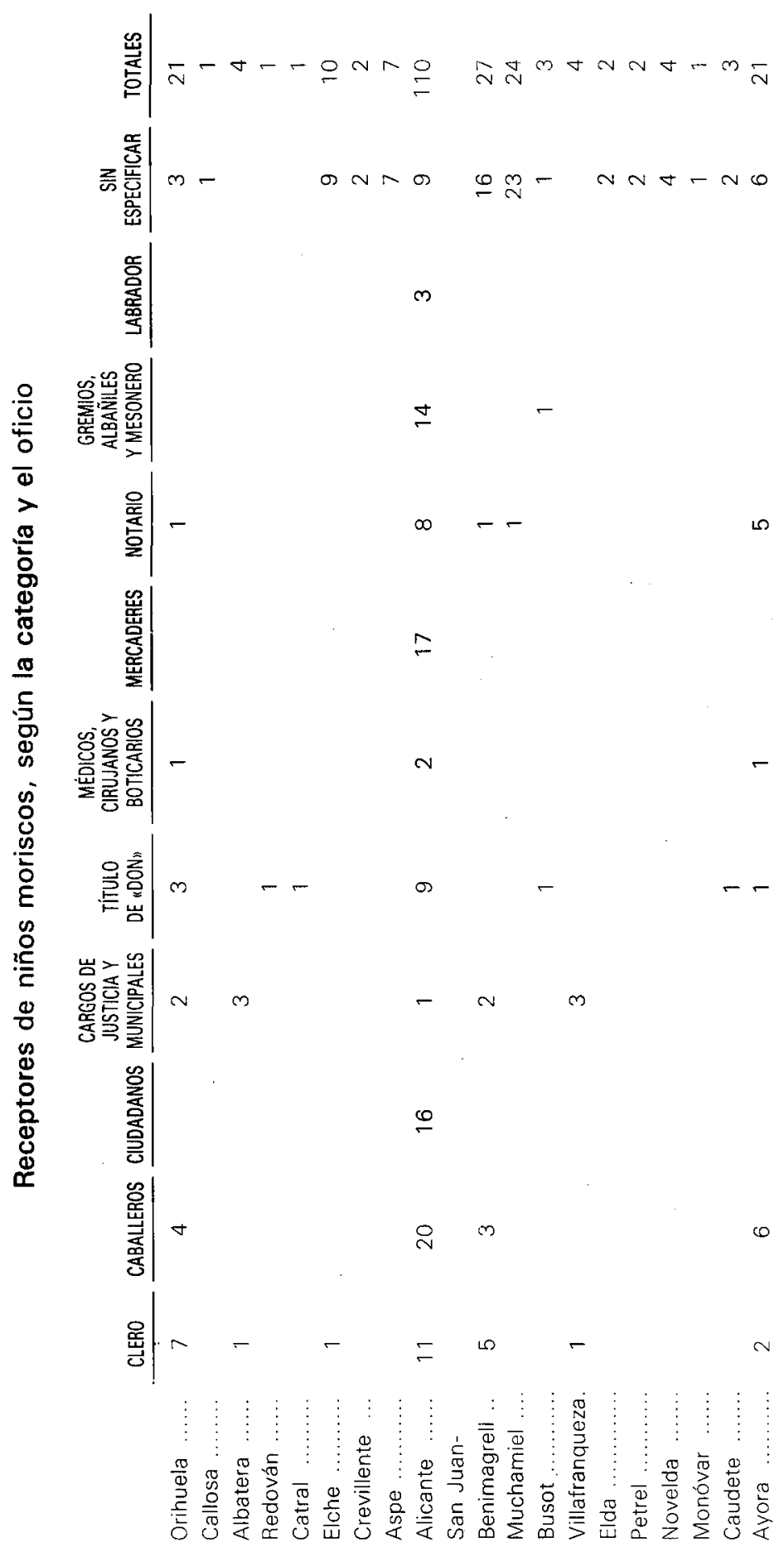
Es evidente la apropiación de los niños por personas de relieve social en Orihuela, Albatera, Redován, Catral, Alicante, Villafranqueza, Caudete y Ayora. En las otras poblaciones no queda tan claro que los niños moriscos fueran encomendados a los grupos de más elevado rango social. Por ejemplo, en Elche, salvo el caso de una niña de 8 meses que está en la casa de mosén Antonio Tarí, los otros 9 se asignan a casas cuyo cabeza de familia aparece sin título de "don" ni otro dato indicativo de su posición social o cargo. Resulta difícil pensar que estas personas estuviesen integradas en los grupos sociales privilegiados al suprimir en todos los casos el título o la categoría social de cada uno de ellos, y más en un siglo en el que las categorías sociales y las distinciones en función de ellas se cotizaban tanto.

El reparto de los niños moriscos de Alicante entre las diversas familias de la ciudad ha sido analizado también por M. Martínez Gomis, con una clasificación socio-profesional distinta de la que aparece en el cuadro que aquí presento. M. Martínez incluye, siguiendo a R. Benítez, las actividades mercantiles con las artesanales en "oficios mecánicos y viles", y da la exigua cifra de 5 niños acogidos por estos profesionales. Incluso añade que la diferencia entre Málaga, donde sí había gran número de niños moriscos encomendados en este grupo, y Alicante "puede deberse a que la inmensa mayoría de los mercaderes instalados en Alicante a principios del S. XVII eran extranjeros y a que, por tanto, no intervinieron en los hechos de Laguart, quedando ausentes del reparto del botín» (11).

En la Relación del obispo Balaguer hay 17 mercaderes alicantinos con niños moriscos; si se agrupan con los receptores de profesiones gremiales o artesanales, mesoneros y albañiles, el porcentaje de familias no privilegiadas con niños moriscos resulta muy superior al que se registra en el trabajo anteriormente citado.

Esta circunstancia es extensible a todo el obispado si mantenemos la hipótesis de que aquellas familias de las que no se especifica título ni grupo social, pertenecen a grupos no privilegiados. Los cabezas de familia con niños moriscos a su cargo, que no se inscriben con título de "don» ni ninguna otra calificación, suponen el $35,5 \%$.

Por tanto, aún reconociendo que los grupos sociales privilegiados reciben niños moriscos en una proporción alta, no es desdeñable el porcentaje que alcanzan los grupos menos privilegiados de la sociedad como receptores de moriscos.

Estos niños moriscos representan el $81 \%$ de todos los moriscos que se quedaron en el obispado de Orihuela después de la expulsión, según la fuente que analizamos.

El obispo que la confecciona menciona otros grupos, de los que el más

(11) Ibidem, p. 274. 
numeroso es el formado por quienes recibieron licencia para quedarse por haber dado muestras de estar asimilados antes de la expulsión. Suponen el $15,3 \%$ de los moriscos que se quedaron en la diócesis oriolana.

Pueden dividirse a su vez en:

1. Los que conservan casa propia.

2. Los que se integran en familias cristianas viejas.

Constituyen el primer grupo once familias distribuidas en ocho lugares, con treinta y dos miembros. Sólo hay cuatro matrimonios completos; el resto lo integran un viudo, dos viudas, tres varones con hijos de los que se ignora la condición e identidad de la esposa y una madre cuyo marido se había embarcado (12).

El grupo de moriscos mayores de edad que se integra en familias cristianas es todavía más exiguo: ocho hombres - dos de ellos casados, uno con cristiana vieja que tiene tres hijos y una nieta-, dos mujeres y una viuda, repartidos por seis poblaciones del obispado; entre todos alcanzan la cifra de dieciséis moriscos (13).

Nada se indica acerca del grado de asimilación que se había observado en ellos antes de la expulsión. Sólo se menciona que dos mujeres de 25 y 20 años se convirtieron en el año del edicto de gracia (14).

En tres casos se refiere que uno de los cónyuges se embarcó y los otros permanecieron en España (15).

Los restantes grupos de moriscos que permanecieron en la diócesis tras la expulsión reultan insignificantes. En primer lugar, están aquellos que han probado ser hijos de cristianos viejos, entre los que se incluye, junto a tres muchachos, a una viuda berberisca de 80 años y un morisco casado con cristiana vieja, con dos hijos (16).

Finalmente, cuatro moriscas, esposas o viudas de cristianos viejos, dos de ellas con descendencia (17).

En consecuencia, el número de los moriscos que permenecieron en el obispado oriolano, según esta fuente, es muy pequeño respecto a la antigua

\footnotetext{
(12) Ver Apéndice documental.

(13) Ver Apéndice documental.

(14) Es posible que se refiera a la predicación de 1606, y no a la de 1599.

(15) En casa del señor de Albatera se quedaron dos moriscos albaterenses de 50 y 53 años cuyas respectivas mujeres se habian embarcado, y otro tanto sucedió con una mujer en Orihuela.

(16) Ver Apéndice documental.

(17) Ver Apéndice documental.
} 
población morisca, y todavía más insignificante con relación a los cristianos viejos.

El odio y las tensiones sociales entre los dos linajes eran máximos en el reino valenciano, y fue el morisco levantino el que provocó la expulsión y le confirió un carácter radical. El mismo empeño en que los niños moriscos residieran en el interior, a tres leguas de la costa al menos, hay que tomarlo como una rémora del pasado. Nada había que temer de unos niños aislados, apartados de la tradición de sus mayores e integrados en familias cristianas.

Otro tanto sucedía con los escasos moriscos mayores de edad que permanecieron en casas de cristianos viejos; incluso los que conservaban casa propia carecían de la comunidad, que era el medio en el que pervivían sus tradiciones y creencias.

Con la desaparición de la comunidad morisca los escasos cristianos nuevos que se quedaron en el obispado de Orihuela se integraron inmediata y definitivamente en la comunidad cristiana. 


\section{APÉNDICE DOCUMENTAL}

He seleccionado la parte más breve de la relación del obispo Balaguer, que afecta a tres de los cuatro grupos en que estructura el documento. Se omite, por su mayor extensión, la primera, relativa a los niños moriscos.

A. General de Simancas, Estado, Ig. 224, s. f.

"Los que quedan en el obispado con licencia del obispo, por haver dado muestras de christianos antes de la expulsión.

\section{Orihuela}

- En casa de mosén Francisco Martínez, cura de Santiago, Pedro Pérez, de 40 años; es de Albatera.

- En casa propia, Gerónima Monçona, de 35 años; es de Redován, embarcose su marido; hijo Luis Martínez, de 18 años; es de Orihuela.

- En casa de D. Juan Rocamora, señor de La Granja, Jayme Ruiz, de 30 años; es de La Granja.

- En casa de Ginés Almodóvar, cavallero, Pedro Vayo, de 40 años; es de Redován; casó con christiana vieja. Hijo Pedro, de 4 años; hijo Juan de 6 meses; hija María, viuda, de 25 años; hija Isabel, de 2 años.

- En casa de Jayme Ruiz, cavallero, Leonor Despuig, de 25 años, es de Monnovar, se convirtió en la predicación del edicto de gracia.

- En casa de D. Baltasar Rocamora, Isabel Despuig, de 20 años, es de Monovar, se convirtió el año del edicto de gracia.

- En casa propia, la viuda de García, de 60 años; hija Isabel, de 8 años; hija María, de 22 años, casada con christiano viejo.

\section{Almoradí}

- En casa de Francisco Plorejant, labrador, Isabel Ortigosa, de 55 años, viuda; hija María, de 8 años.

\section{La Granja}

- En casa propia, Miguel Mandado, de 60 años; hijo Miguel, de 25 años; hijo Juan, de 16 años.

- En casa propia, Pedro Mandado, de 30 años; hijo Miguel, de 6 años; hijo Pedro, de 8 meses.

\section{Albatera}

- En casa de D. Ramón de Rocafull, señor de dicha villa, Henrrique Mexijo, de 30 años, es de Albatera, embarcose su mujer.

- En casa propia, Diego López, de 40 años. Su mujer Águeda Aguilara, de 35 años; hijo Alonso, de 15 años; hijo Luis, de 2 años; hija Ángela, de 8 años; hija Cathalina, de 5 años; cuñado Luis Aguilar, de 20 años. 


\section{Crevillente}

- En casa propia, Juan Sala, viudo, de 40 años, es de Crevillente; hija Ángela, de 10 años; hijo Juan, de 8 años; hija María, de 6 años.

Aspe

- En casa propia, Agustín Portillo, de 30 años; hijo Jusepe, de 6 años; está en casa de Jayme Montero.

\section{Elda}

- En casa propia, Francisco Alpal, de 60 años, es de Elda; su mujer Leonor Merita, de 50 años; cuñada sorda y ciega de 54 años.

- En casa de Juan Olivares, Jusepe Cucuma, de 17 años, es de Elda.

Novelda

- En casa de D. Francisco Maça, Miguel Mandado, de 26 años, es de La Granja.

\section{Monnovar}

- En casa de Juan Despuig, Francisco Jebrer, de 40 años, es de Monnovar; su mujer Isabel Champa, de 40 años, es de Elche.

- En casa propia, Martín Pérez Morón, de 34 años, es de Novelda. Su muger Jusepa Corroy, de 25 años, es de Monnovar.

\section{Ayora}

- En casa propia, Francisca Christiana, viuda de 56 años, es de Jeresa, se convirtió el año de la predicación, y siempre después ha vivido en la villa de Ayora.

Los que han provado ser hijos de christianos viejos y morisca, y pretenden no estar comprendidos en los bandos de la expuisión y tienen también licencia del Obispo.

\section{Origuela}

- En casa del Obispo, Francisco Martínez, de 14 años, es de Origuela; quando su padrastro se embarcó se fue huyendo a casa del Obispo.

- En casa del Dr. Juan Ferrández de Mesa, Juana Jaso, de 18 años.

- En casa del Dr. Luis Ocaña, Juana Pérez, de 20 años, es de Novelda.

Elche

- La viuda Magara, berberisca, de 80 años.

Elda

- En casa propia, Francisco Murillo, de 60 años; su mujer es christiana vieja, hijo Francisco, de 20 años; hijo Pedro, de 16 años. 
Las mujeres moriscas que están casadas con christianos viejos o han quedado viudas.

\section{Origuela}

- Cathalina Geroni, viuda de Juan Carrança, cavallero christiano viejo, de Novelda; agora es beata de S. Francisco.

Elda

- En casa propia, Juana Morisca de 60 años, mujer de Juan Gonzáles, christiano viejo; tiene hijos y nietos y todos son buenos christianos.

- En casa de Juan Carrança, christiano viejo, su mujer Jal Carrança, de 40 años, criada de pequeña en la casa de los condes de Elda.

\section{Monnovar}

- Isabel Faxarda, mujer de Balthazar Alvado, christiano viejo, de 40 años, tienen tres hijos. 